\title{
Graefe's archive for clinical and experimental ophthalmology—instant glue for retinal detachment surgery?
}

\author{
Ning Cheung • David Wong
}

Received: 19 August 2014 / Accepted: 22 August 2014 / Published online: 7 October 2014

(C) Springer-Verlag Berlin Heidelberg 2014

Retinal tears were first observed in 1853 , soon after the advent of the ophthalmoscope [1]. However, it took half a century before their role in the causation of rhegmatogenous retinal detachment was recognized, based on the seminal work by Jules Gonin from 1904 to 1931 [1]. Using a hot metal probe passing through a scleral incision, Gonin developed the first successful technique for retinal detachment repair by inducing thermal injury to the retina, retinal pigment epithelium (RPE), and choroid adjacent to retinal tears. Subsequent wound healing formed a watertight barrier between subretinal space and vitreous cavity.

To date, thermal injury remains the basis of treatments for all retinal tears in retinal detachment surgery, ranging from Gonin's hot metal probe to penetrating diathermy, and now contemporary cryo- or laser retinopexy. It relies on wound healing to establish sufficiently tight chorioretinal fusion to seal off access of the subretinal space via retinal breaks. This process takes time. Additionally, both the retina and RPE or choroid must be held in apposition, either by indentation of the scleral wall (scleral buckling) or internal tamponade by gas or silicone oil. Chorioretinal adhesion caused by cryo- or laser retinopexy has been reported to take between 2 and 4 weeks to reach maximum strength of adhesion [2, 3]. Nevertheless, laser photocoagulation may achieve a more rapid, albeit limited, enhancement of chorioretinal adhesion (within 1 to

N. Cheung $\cdot$ D. Wong $(\bowtie)$

The Eye Institute, Department of Ophthalmology, University of Hong Kong, Pokfulam, Hong Kong, China

e-mail: shdwong@hku.hk

N. Cheung

Singapore Eye Research Institute, Singapore National Eye Centre, Singapore, Singapore

N. Cheung

Centre for Eye Research Australia, Royal Victorian Eye and Ear

Hospital, University of Melbourne, Melbourne, Australia
3 days), whereas cryotherapy tends to invariably weaken adhesion for up to 1 week before it strengthens adhesion [3]. It has been postulated that the more acutely increased adhesion from laser retinopexy could be related to the development of a proteinaceous coagulum, and the initial reduced adhesion from cryo-retinopexy could be due to chorioretinal edema. Regardless, both techniques rely chiefly on delayed wound healing and scar formation over a matter of weeks to achieve the degree of chorioretinal adhesion that can be considered an adequate barrier to prevent re-detachment of the retina.

In this issue, Umanets and colleagues have examined the potential use of high-frequency electric welding (HFEW) as a new retinopexy technique for retinal detachment surgery [4]. In this experimental study, live rabbit eyes with flat retina were randomly assigned to receive standard laser retinopexy, HFEW retinopexy with two different voltage settings, or no intervention (controls). Enucleation was then performed and a full-thickness fragment of eye-wall tissue containing the area of retinopexy was isolated. Using a customized machine, the tensile strength of the chorioretinal adhesion was measured at various time points. The main finding was that HFEW retinopexy generated significantly firmer, and, more importantly, almost immediate ( $1 \mathrm{~h}$ or less) chorioretinal adhesion than that of laser retinopexy. The results of this study raised several important issues.

Previous investigators have attempted to exploit instant adhesion during retinal surgery. Vicente Martinez-Castillo, for example, performed protracted vitrectomy shaving tightly the vitreous base and draining meticulously the last bit of subretinal fluid (by using an air bubble over perfluorocarbon liquid) [5]. Others have tried and failed with this technique. Perhaps there is a steep learning curve, but for whatever reason, the technique is not widely adopted, and there have been few publications to corroborate and repeat the excellent results published 7 years ago. Nonetheless, as a proof of principle, the publication has been highly influential and is 
often quoted. Interestingly, the author prefers transscleral diode laser retinopexy, which is not readily available to most surgeons. Whether this form of retinopexy could induce stronger and more instant adhesion than standard endolaser retinopexy remains unknown. However, the benefits of not using tamponade are well recognized [5].

It is interesting to speculate why HFEW could create an instant chorioretinal fusion when laser often could not. The investigators suggested that the electric current from HFEW caused intracellular protein denaturation within the outer retinal cell layers, and subsequent accumulation of the denatured proteins in the extracellular matrix "glued" the chorioretinal layers together. Similarly, laser use induces a photocoagulation effect largely through the denaturing of proteins [6]. Perhaps the key difference between HFEW and the laser is the level at which the energy is taken up. Laser photocoagulation relies on RPE cells to absorb the energy, which then "slowly" heats up the overlying outer retinal cells. In contrast, HFEW's electric shock may heat up the retinal cells directly and then almost instantaneously transmit this energy through the RPE and choroidal layers, leading to formation of a stronger and instant denatured proteinaceous coagulum that fuses all the layers together. In addition, it is possible that the technique of applying HFEW may also help achieve instant fusion. Unlike the laser and cryotherapy, HFEW retinopexy requires direct contact of the monopolar electrode probe with the retinal surface. This maneuver would likely indent the retinal surface slightly and bring the outer retinal cell layer closer to the RPE layer. Thus the authors literally used the term "spot welding". Direct cellular contact of these two layers might further facilitate instant fusion upon activation of the electric current.

Obviously, the retinopexy technique, which can achieve instant chorioretinal adhesion, has attractive clinical advantages in retinal detachment surgery. Besides the ability to avoid the inconvenience and complications of postoperative gas or silicone oil tamponades, another important advantage is the possibility to eliminate one major reason for retinal redetachment-missed retinal breaks [7]. After treating all the retinal breaks found intraoperatively, surgeons would be able to know whether there are any missed breaks. If the retina remains attached with balanced-salt-solution only in situ, it would give greater confidence that all breaks responsible for the detachment had been found and treated adequately.
In recent decades, there have been few advances in retinopexy techniques while instant and secure closure of all retinal breaks remains a much desirable clinical endpoint for retinal detachment surgery. Despite some limitations (e.g., animal experiments done in non-vitrectomized eyes with attached retina), the positive findings of the study by Umanets and colleagues offer a sense of excitement for a new promising procedure that sheds light into achieving this goal in the future. As the investigators hinted, much work is still ongoing. It therefore remains to be seen whether HFEW retinopexy will truly become a game-changer leading to a paradigm shift in the management of rhegmatogenous retinal detachment.

Conflict of interest All authors certify that they have no affiliations with or involvement in any organization or entity with any financial interest (such as honoraria; educational grants; participation in speakers' bureaus; membership, employment, consultancies, stock ownership, or other equity interest; and expert testimony or patent-licensing arrangements), or non-financial interest (such as personal or professional relationships, affiliations, knowledge or beliefs) in the subject matter or materials discussed in this manuscript.

\section{References}

1. Gloor BP, Marmor MF (2013) Controversy over the etiology and therapy of retinal detachment: the struggles of Jules Gonin. Surv Ophthalmol 58(2):184-195

2. Yoon YH, Marmor MF (1988) Rapid enhancement of retinal adhesion by laser photocoagulation. Ophthalmology 95(10):1385-1388

3. Kita M, Negi A, Kawano S, Honda Y (1991) Photothermal, cryogenic, and diathermic effects of retinal adhesive force in vivo. Retina 11(4): 441-444

4. Umanets N, Pasyechnikova NV, Naumenko VA, Henrich PB (2014) High-frequency electric welding: a novel method for improved immediate chorioretinal adhesion in vitreoretinal surgery. Ophthalmol, Graefes Arch Clin Exp

5. Martinez-Castillo V, Zapata MA, Boixadera A, Fonollosa A, GarciaArumi J (2007) Pars plana vitrectomy, laser retinopexy, and aqueous tamponade for pseudophakic rhegmatogenous retinal detachment. Ophthalmology 114(2):297-302

6. Tababat-Khani P, Berglund LM, Agardh CD, Gomez MF, Agardh E (2013) Photocoagulation of human retinal pigment epithelial cells in vitro: evaluation of necrosis, apoptosis, cell migration, cell proliferation and expression of tissue repairing and cytoprotective genes. PLoS One 8(8):e70465

7. Lois N, Wong D (2013) Complications of vitreoretinal surgery: Lippincott Williams \& Wilkins 